Catatonia: the tension insanity

SIR: Johnson in his lecture article (Journal, June $1993,162,733-738$ ) suggests that catatonia should be regarded as a neuropsychiatric syndrome in which an abnormal mental state is associated with 'cataleptic phenomena'. Cataleptic phenomena, as used by Johnson, essentially refer to features of catatonic stupor, with immobility, maintenance of posture and mutism constituting a diagnostic triad. Such a view of catatonia would virtually exclude those 'catatonic' conditions in which only the excited state is present.

Catatonia is generally regarded as having two phases - retardation (stupor) and excitement - with sometimes rapid alternation between the two. Although less common, the excited form may be the only state present. Morrison (1973) studied 250 cases of catatonia and found that 110 were predominantly retarded, 67 predominantly excited, and the remainder in a mixed state. It is interesting to note that the three-state concept of catatonia - retarded, excited and mixed - is incorporated into the ICD-10 diagnostic guidelines (World Health Organization, 1992) for a new category: organic catatonic disorder.

Morrison's study also showed that retarded and excited patients differed not only in obvious presentation but also in course and prognosis. Retarded patients were significantly more often negativistic, mute, rigid and cataleptic - features corresponding to Johnson's cataleptic phenomena - whereas excited patients were more frequently impulsive, combative and denudative. Excited patients were also characterised by an abrupt, rapid onset, were more likely to be greatly improved at discharge, and were more often recovered at follow-up. Moreover, they were more frequently diagnosable as having affective disorder, mostly mania.

Nevertheless, considerable symptom overlap was also noted between the two subtypes. No one symptom was limited to one type, and of those special symptoms supposedly 'typical' of catatonia, only four-mutism, negativism, posturing and staring into space - were present in half or more of the total patients. Such symptom overlap, coupled with the fact that the two states may co-exist or alternate rapidly, would argue against separating them as two distinct syndromes. They are best considered distinct subtypes subsumed under the catatonic syndrome.

The past 20 years have seen no comparable largescale symptomatology studies. Research attention to the two subtypes of catatonia has been scanty. No distinction is often made between the two states, or interest is concentrated only on the retarded form. There have been a number of reports in recent years on the use of drugs such as diazepam (McEvoy \& Lohr, 1984), lorazepam (Salam et al, 1987) and carba- mazapine (Rankel \& Rankel, 1988) in the treatment of catatonia (psychogenic- or neuroleptic-induced), but they are all for the stuporous state. A re-evaluation of the excited form of the syndrome as a catatonic subtype is indicated. To remove it from the catatonic syndrome at the present stage would appear unjustified.

McEvoY, J. P. \& LoHR, J. B. (1984) Diazepam for catatonia. American Journal of Psychiatry, 141, 284-285.

MoRRIson, J. R.(1973)Catatonia: retarded and excited types. Archives of General Psychiatry, 28, 39-41.

RANKEL, H. W. \& RANKEL, L. E. (1988) Carbamazepine in the treatment of catatonia. American Journal of Psychiatry, 145, 361-362.

Salam, S. A., Pillai, A. K. \& Beresford, T. P. (1987) Lorazepam for psychogenic catatonia. American Journal of Psychiatry, 144, 1082-1083.

WORLD Health Organization (1992) The ICD-10 Classification of Mental and Behavioural Disorders. Geneva: WHO.

Tokanui Hospital

WAI-YU JoSEPH

Private Bag

Te Awamutu

New Zealand

\section{Low serum cholesterol and suicide}

SIR: Hawton \& Cowen (Journal, June 1993, 162, 818$825)$ consider the suggestion that the link between low cholesterol and suicide is secondary to the reduced appetite and weight loss which may occur with depressive illness. In an attempt to clarify the links between cholesterol and depression, we studied six depressed men over the age of $\mathbf{4 0}$ with no history of ischaemic heart disease (IHD), before treatment and on recovery, and six age-matched male controls for whom blood was obtained at the same interval.

Depression is associated with an increased risk of death from IHD (Rabins et al, 1985) and one might therefore expect that patients with depressive illness would have high plasma cholesterol, high low-density lipoprotein cholesterol (LDL-C), low high-density lipoprotein cholesterol (HDL-C), or altered HDL subfraction distribution (Griffin et al, 1988).

In our study, there was a non-significant fall in total plasma cholesterol in the depressed group on recovery (mean \pm s.d.: $203.5 \pm 34.9$ v. $175.4 \pm$ $25.9 \mathrm{mg} / 100 \mathrm{ml}$ ) and there was a significant fall in LDL-C in recovered depressive patients (mean \pm s.d.: $102.1 \pm 18.1$ v. $77.4 \pm 17.6 \mathrm{mg} / 100 \mathrm{ml} ; P=$ 0.036 ). There is thus some support for the hypothesis that recovery from depression is associated with changes in circulating cholesterol concentrations, though whether these would be independent of, or central to the changes which result in clinical recovery remains to be established. 